\title{
The unwieldy design of electronic medical records
}

\section{Opinion}

It is approaching 40years since the introduction of a laboratory information management system with then special software for its implementation. There has been enormous progress in the development of the software needed to meet the needs of the large body of data generated by the laboratory, and in likewise, the development of radiology, cardiology, and pharmacy intelligence systems. Moreover, to be effective they all have to be available and readily usable to the attending physicians, nursing and dietary staff. This has led to integrating specially designed data sites with a common electronic medical record that is accessed by the providers of care, and that also protects invasion of the medical record. However, what I have identified is a system or network of systems that has been designed most decidedly for billing purposes, and these are still inadequate at present, despite the enormous costs they incur, and despite the fact that a system may be applied to a hospital and clinic network.

The argument might be made that the information management that contributes to increased cost of care improves the quality of care, which remains to be proved. The potential for better quality surveillance is built in. However, patient care requires adequate human interaction. In order to maintain adequate staffing levels, there is a growing shortage of nurses and their replacement by paramedical staffing. This might be workable if the right balance of staffing to patients is maintained. However, the most serious problem related to staffing and workloads is the excessive time that the provider is required to enter and extract information from the data. All this is not necessary if the computer is designed, integrated, and has computational intelligence. In the cardiology and intensive care components the design might be suitable for the activities involved. But the activities carried out are intensive, so the design must have a quality component that follows the blood gases and other critical measures sufficiently well. There was a time past that there was a separate pulmonology laboratory adjacent to the OR or ICU that handled the blood gases in a time frame that the central laboratory could not meet. The laboratory is actually a group of laboratories that prepare and interpret biopsies, take and prepare blood, analyze the blood for chemistry, hematology, microbiology, blood bank, and perhaps special procedures such as, genomics or proteomics. The
Volume 5 Issue 3 - 2017

\author{
Larry H Bernstein \\ Triplex Medical Diagnostics, USA
}

Correspondence: Larry H Bernstein, Triplex Medical Diagnostics, 54 Firethorn Lane Northampton, MA 01060, USA, Email larry.bernstein@gmail.com

Received: November 20, 2017 | Published: December 0I, 2017

construction of the laboratory is such that the reports are maintained in separate frames, as if they were unrelated results. The time has passed for such a representation of data. Furthermore, the analyses that are carried out have contextual meaning. As a result, the technologists who are processing the specimens might well have criteria by which to decide whether the test is significant, technically in error, or indicates the performance of another test. This is more complicated than it seems because any additional testing has to be ordered by a physician. If necessary to meet that criterion, the information system should inform the physician without delay, and the laboratory would also be enabled to set aside a specimen for further analysis.

This also brings to mind the issue of billing and of correct diagnosis. Of course there are billing codes. The information system should have the computational ability to determine the probability for the selected diagnoses, and the related billing code. Only if we proceed forward in a more clinically expressed manner will we resolve a crisis in the making.

\section{Acknowledgements}

None.

\section{Conflict of interest}

The author declares no conflict of interest. 